\title{
Resonance Tunneling in Ferromagnet-Triplet Superconductors Junctions
}

\section{Kowalewski, R.J. Wojciechowski and P. Wojtuś}

A. Mickiewicz University, Institute of Physics

Umultowska 85, 61-614 Poznań, Poland

\begin{abstract}
We discuss the tunneling conductance in a ferromagnet-insulator-triplet superconductor junction. We consider the superconducting order parameters with spin triplet pairing having nodes. The nodal structure of the order parameter has been recently confirmed experimentally in $\mathrm{Sr}_{2} \mathrm{RuO}_{4}$. In particular, we study how a mid-gap structure of the tunnelling conductance depends on the phase difference of the pairing potential as well as on the orientation of the interface.
\end{abstract}

PACS numbers: 74.70.Pq, 73.40.-c, 74.45.+c

\section{Introduction}

The nature and symmetry of the spin-triplet superconductivity in $\mathrm{Sr}_{2} \mathrm{RuO}_{4}$ is still a subject of both theoretical and experimental investigation (e.g. [1-7] and references therein). The reason for the interest in this subject is that an orbitally anisotropic pairing state is still not well determined.

As the number of possible triplet pairing states is very large, it still remains an open question, which of them is really relevant for $\mathrm{Sr}_{2} \mathrm{RuO}_{4}$. The power-law behaviour of the temperature dependence of the specific heat and the nuclear spin-lattice relaxation rate of ${ }^{101} \mathrm{Ru}$ in $\mathrm{Sr}_{2} \mathrm{RuO}_{4}$ suggest the existence of lines of nodes in the pairing states. However, the positions of these lines are still not established and further research of this problem is necessary.

The objective of this paper is to discuss the spin polarized charge tunnelling transport in a ferromagnet-insulator-triplet superconductor junction (F/I/TS), and, in particular, at bias voltage smaller than the gap function. These tunneling effects are sensitive both to a phase difference of pair potentials (contained in electron- and hole-like quasiparticle spectrum) and to the orientation of the interface with respect to the $a$-axis of $\mathrm{Sr}_{2} \mathrm{RuO}_{4}$ crystal (see Fig. 1). It seems that the tunneling effects can provide the most precise information on the symmetry 


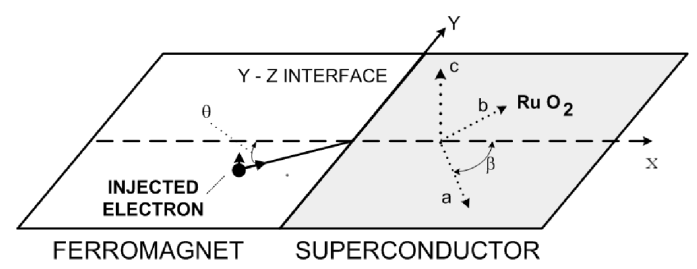

Fig. 1. Schematic illustration of $Y-Z$ junction. $\Theta$ and $\beta$ denote the angles of injection of the electron and the orientation of the interface, respectively.

of the superconducting order parameter. In this paper we use the spin polarized Andreev reflection method to determine the spectra of the tunneling conductance in $\mathrm{F} / \mathrm{I} / \mathrm{TS}$ junctions.

\section{Bogolyubov-de Gennes equations and tunneling conductance}

The energy and wave function of quasiparticles, which are injected, reflected and transmitted through a F/I/TS junction, can be obtained from the extended Bogolyubov-de Gennes (BdG) equations, valid for both sides of the junction

$$
\begin{aligned}
& \left(\begin{array}{cc}
\left(H_{0}-E\right) \check{1}-U \check{\sigma}_{z} \Theta(-x), & \check{\Delta}\left(\Theta_{s}, x\right) \Theta(x) \\
\check{\Delta}^{\dagger}\left(\Theta_{s}, x\right) \Theta(x) & -\left[\left(H_{0}-E\right) \check{1}-U(x) \check{\sigma}_{z} \Theta(-x)\right]^{\dagger}
\end{array}\right)\left(\begin{array}{l}
u_{\uparrow}(\boldsymbol{r}) \\
u_{\downarrow}(\boldsymbol{r}) \\
v_{\uparrow}(\boldsymbol{r}) \\
v_{\downarrow}(\boldsymbol{r})
\end{array}\right) \\
& \quad=\check{O}
\end{aligned}
$$

where $H_{0}$ is the single-particle Hamiltonian $H_{0}=-\hbar^{2} \nabla^{2} / 2 m+\left(V-U_{\mathrm{B}} \check{\sigma}_{z}\right) \delta(x)-$ $E_{\mathrm{F}}, E$ - energy of the quasiparticle, $\check{\sigma}_{z}$ - Pauli matrix, $\check{1}$ - unity matrix, $\Theta(x)$ - Heaviside step function, $V$ - barrier height, and $U_{\mathrm{B}}$ - the exchange energy in the barrier. We choose the $z-y$ interface and tunneling along the $x$ axis in the $\mathrm{RuO}_{2}$ plane. The gap function $\Delta$ can be written in the following matrix form:

$$
\begin{gathered}
\check{\Delta}= \\
\mathrm{i} \sigma_{y}(\boldsymbol{d}(\boldsymbol{k}) \cdot \boldsymbol{\sigma})=\left[\begin{array}{c}
d_{x}+\mathrm{i} d_{y},-d_{z} \\
-d_{z},-d_{x}+\mathrm{i} d_{y}
\end{array}\right] \\
=\left[\begin{array}{cc}
\Delta_{\uparrow \uparrow}\left(\Theta_{s}, x\right), & \Delta_{\uparrow \downarrow}\left(\Theta_{s}, x\right) \\
\Delta_{\downarrow \uparrow}\left(\Theta_{s}, x\right), & \Delta_{\downarrow \downarrow}\left(\Theta_{s}, x\right)
\end{array}\right],
\end{gathered}
$$

where $\Theta_{s}$ and $x$ denote directions of motion of the quasi-particles and the centre of mass of a Cooper pair, respectively. Let us assume the quantization axis of the spin triplet superconductor perpendicular to the superconducting $\mathrm{RuO}_{2}$-plane.

In general, several different tunnelling and reflection processes may co-exist at the interface of the F/I/TS junction. These processes are determined by many factors such as position of the Fermi energy on both sides of the junction, strength of the exchange potential, strength of the barrier potential, spin polarization of the injected electrons, and orientation of the interface. For unitary states, the spin-up electrons can be paired with the spin-down electrons forming orbitally 
anisotropic Cooper pairs. Therefore, the spectral analysis of the spin polarized tunnelling conductance can be used to identify the anisotropic order parameters of the superconductor. The tunneling charge conductance depends not only on symmetry of the pairs and strength of the exchange field, but also, on the barrier potentials, which are different for spin-up and spin-down particles.

We analyze the charge conductance spectra in $\mathrm{F} / \mathrm{I} / \mathrm{TS}$ junctions with respect to the strength of molecular field in a ferromagnet, various barrier potentials at the interface, and several orientations of the interface. The spin dependent BdG equations for the assumed superconducting pairing states can be decoupled into two mutually independent sets of two component equations. The wave function $\Psi(x)$ at the ferromagnetic side of the junction is a sum of the wave functions of the incoming electron with up or down spin $(\uparrow(\downarrow))$, the reflected hole and reflected normal electron with up or down spin. In the case of unitary pairing state, the incoming electron and the Andreev reflected hole have antiparallel spins, while in the case of nonunitary Cooper pairs, the Andreev reflected hole conserves the spin of the injected electron.

Therefore, the wave function, at the ferromagnetic side of the junction, for unitary $\left(\Psi_{\uparrow(\downarrow)}^{\mathrm{u}}\right)$ states can be written in the following form:

$$
\begin{aligned}
& \Psi_{\uparrow(\downarrow)}^{\mathrm{u}}(x)=\left(\begin{array}{l}
1 \\
0
\end{array}\right) \exp \left(\mathrm{i} k_{\uparrow(\downarrow)} x \cos \Theta\right)+a_{\downarrow(\uparrow)}\left(\begin{array}{l}
0 \\
1
\end{array}\right) \exp \left(\mathrm{i} k_{\downarrow(\uparrow)} x \cos \Theta_{\mathrm{A}}\right) \\
& +b_{\uparrow(\downarrow)}\left(\begin{array}{l}
1 \\
0
\end{array}\right) \exp \left(-\mathrm{i} k_{\uparrow(\downarrow)} x \cos \Theta\right),
\end{aligned}
$$

where $k_{\uparrow(\downarrow)}=k_{\mathrm{F}} \sqrt{1 \pm x}, x=U / E_{\mathrm{F}} . \Theta_{\mathrm{A}}$ denotes the angle of reflection of the Andreev hole. The wave function $\Psi^{s}(x)$ on the superconducting side is a sum of electron-like and hole-like quasi-particles

$$
\begin{aligned}
& \Psi(x)=c_{\uparrow}\left(\begin{array}{c}
u\left(\Theta_{s}\right) \\
\phi_{+}^{*} v\left(\Theta_{s}\right)
\end{array}\right) \exp \left(\mathrm{i} k_{s} x \cos \Theta_{s}\right) \\
& \quad+d_{\uparrow}\left(\begin{array}{c}
\phi_{-} v\left(\pi-\Theta_{s}\right) \\
u\left(\pi-\Theta_{s}\right)
\end{array}\right) \exp \left(-\mathrm{i} k_{s} x \cos \Theta_{s}\right),
\end{aligned}
$$

where $k_{s}=k_{\mathrm{F}}$ is assumed. The phase factor $\phi_{ \pm}=\exp \left(\mathrm{i} \varphi_{ \pm}\right)$. The coherence factors are $u\left(\Theta_{s}\right)$ and $v\left(\Theta_{s}\right)$, respectively.

The angles of incidence, reflection, and transmission of the quasi-particles are related to one another by the Snell law. It is easy to show that in the case of unitary state there are two critical angles of incidence $\Theta_{\mathrm{c} 1}$ and $\Theta_{\mathrm{c} 2}$, which divide the region of the incident angle into the following three different regions: $0<\Theta<\Theta_{\mathrm{c} 2}, \Theta_{\mathrm{c} 2}<\Theta<\Theta_{\mathrm{c} 1}$, and $\Theta>\Theta_{\mathrm{c} 1}$. When $\Theta>\Theta_{\mathrm{c} 1} \equiv \sin ^{-1}\left(k_{s} / k_{\mathrm{F} \uparrow}\right)\left(k_{s}<k_{\mathrm{F} \uparrow}\right)$ the total normal reflection occurs and the charge and spin currents from the ferromagnet to the superconductor vanish. Thus, the probability of the normal reflection is equal to 1 . On the other hand, when $\Theta_{\mathrm{c} 1}>\Theta>\Theta_{\mathrm{c} 2} \equiv \sin ^{-1}\left(k_{\mathrm{F} \downarrow} / k_{\mathrm{F} \uparrow}\right)\left(k_{\mathrm{F} \downarrow}<k_{s}<k_{\mathrm{F} \uparrow}\right)$ the $x$ 
component of wave vector is purely imaginary. The Andreev reflected holes do not propagate but a finite amplitude of the Andreev reflected process still exists $\left(\left|a_{\uparrow}(E, \Theta)\right|^{2}>0\right)$. In this virtual Andreev reflection region the charge and spin currents from the ferromagnet to the superconductor still exist. In the case of non-unitary states one critical angle $\Theta_{\mathrm{c} 1}$ exists. When $\Theta<\Theta_{\mathrm{c} 2}$ (for unitary states) or when $\Theta<\Theta_{c 1}$ (for non-unitary states) the Andreev holes exist and propagate in the ferromagnet. The amplitudes for both Andreev and normal reflections contain the term $\delta\left(E, \Theta_{s}, \varphi_{+}-\varphi_{-}\right)[7]$ which is very sensitive to the phase difference $\varphi_{+}-\varphi_{-}$of the pairing potential felt by the electron-like (ELQ) and hole-like (HLQ) quasi-particles as well as to the angle of the interface orientation $\beta$. This sensitivity especially reveals itself in

$$
\delta\left(E, \Theta_{s}, \varphi_{+}-\varphi_{-}\right)=\frac{u\left(\Theta_{s}\right) u\left(\pi-\Theta_{s}\right)-v\left(\Theta_{s}\right) v\left(\pi-\Theta_{s}\right) \phi_{+}^{*} \phi_{-}}{u\left(\Theta_{s}\right) u\left(\pi-\Theta_{s}\right)},
$$

where $\Theta_{s}=\arcsin (\sqrt{1+x} \sin \Theta), \quad \Theta_{\mathrm{A}}=\arcsin \left(\sqrt{\frac{1+x}{1-x}} \sin \Theta\right), \quad$ and $\Delta\left(\Theta_{s}\right) /\left|\Delta\left(\Theta_{s}\right)\right|=\exp \left(\mathrm{i} \varphi_{+}\right)=\phi_{+}, \Delta\left(\pi-\Theta_{s}\right) /\left|\Delta\left(\pi-\Theta_{s}\right)\right|=\exp \left(\mathrm{i} \varphi_{-}\right)=\phi_{-}$. Resonance of the Andreev reflection occurs for certain values of sub-gap energy and angle of the incidence, which fulfil the condition $\delta\left(E, \Theta_{s}, \varphi_{+}-\varphi_{-}\right)=0$.

According to the Blonder-Tinkham-Klapwijk (BTK) formula [8], the intensity of tunnelling conductance depends on the Andreev hole reflection. The reflection is associated with the creation of the ELQ and HLQ. When the energy of the injected electron $E$ is larger than the pairing energy, then ELQ and HLQ can propagate in the superconductor. However, when $E$ is smaller than the gap function, then ELQ and HLQ are bounded at the interface. These bound states are sensitive to the phase difference resulting from the different pair potentials as felt by ELQ and HLQ, and to orientation of the interface determined by the angle $\beta$. The bound states are responsible for conductance peaks which can be interpreted as states created by elastic Andreev retro-reflective scattering on the interface.

Let us discuss the energy spectrum of the tunneling conductance through the F/I/TS junction interface in $y-z$ configuration (see Fig. 1). For simplicity, we discuss the following unitary states with line nodes [2]:

1. $2 \mathrm{D} f$-wave state $B_{1 \mathrm{~g}} \times E_{\mathrm{u}}$ : $\Delta(\Theta)=\Delta_{0} \cos 2(\Theta-\beta)[\cos (\Theta-\beta)+\mathrm{i} \sin (\Theta-\beta)]$,

2. $2 \mathrm{D} f$-wave state $B_{2 \mathrm{~g}} \times E_{\mathrm{u}}$ :

$\Delta(\Theta)=\Delta_{0} \sin 2(\Theta-\beta)[\cos (\Theta-\beta)+\mathrm{i} \sin (\Theta-\beta)]$.

$\beta$ denotes the angle between the normal to the interface ( $x$-axis) and $a$-axis of the crystal in $\mathrm{RuO}_{2}$ plane. The charge conductance though the $\mathrm{F} / \mathrm{I} / \mathrm{TS}$ junction for sub-gap energy $E$ and incident angle $\Theta$ is given by

$$
\sigma_{\boldsymbol{k}_{\uparrow(\downarrow)}}(E, \Theta)=\Re\left(1+\frac{k_{\downarrow(\uparrow)} \cos \Theta_{A}}{k_{\uparrow(\downarrow)} \cos \Theta}\left|a_{\uparrow[\downarrow]}\right|^{2}-\left|b_{\uparrow[\downarrow]}\right|^{2}\right) .
$$

The total tunnelling conductance, divided by that in the normal metal $\left(R_{\mathrm{N}}\right)$ can 
be written as follows:

$$
\sigma_{\boldsymbol{k}}(E)=\sigma_{\boldsymbol{k} \uparrow}(E)+\sigma_{\boldsymbol{k} \downarrow}(E),
$$

where

$$
\sigma_{\boldsymbol{k} \uparrow(\downarrow)}(E)=\frac{1}{R_{\mathrm{N}}} \int_{-\pi / 2}^{+\pi / 2} \mathrm{~d} \Theta \cos (\Theta) \sigma_{\boldsymbol{k}_{\uparrow(\downarrow)}}(E, \Theta) P_{\uparrow(\downarrow)}\left|\boldsymbol{k}_{\uparrow(\downarrow)}\right|
$$

and where $P_{\uparrow(\downarrow)}=(1 \pm x) / 2$.

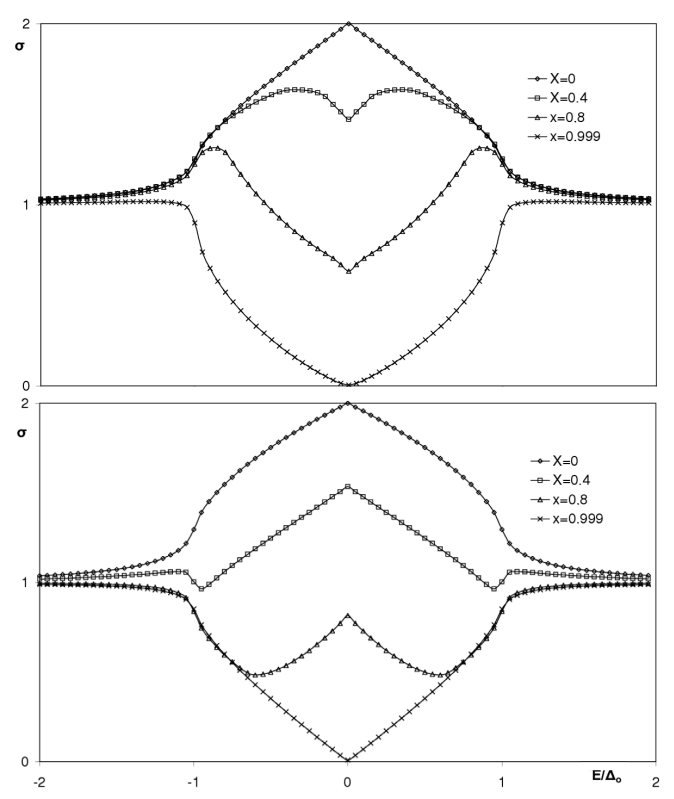

Fig. 2. The normalized tunneling conductance $\mathrm{F} / \mathrm{I} / \mathrm{TS}$ junction with pairing symmetry $B_{1 \mathrm{~g}} \times E_{\mathrm{u}}, z=0$ and $\beta=0$ (the top part) and $B_{1 \mathrm{~g}} \times E_{\mathrm{u}}, z=0$, and $\beta=\pi / 4$ (the bottom part).

The tunneling conductance characteristics strongly depend on orientation of the interface. In Figs. 2-7 there are presented the normalized tunneling conductance spectra $\sigma$ as a function of the normalized energy $E / \Delta_{0}$ for two $2 \mathrm{D}$ $f$-pairing states which have four vertical lines of nodes. The calculations were performed for several values of the angle $\beta$ and the exchange parameter $x$. We have found a qualitatively different behaviour of the conductance spectra for zero and non-zero values of the barrier potential $z$ at the interface. In the case of $z=0$, we observe zero-bias conductance peak for $x=0$ and for arbitrary values of $\beta$. This peak reflects formation of the zero energy states on the interface due to the sign change of the pairing potentials contained in the ELQ and HLQ. The zero energy peak changes into zero energy deep for $x \approx 1$ because of suppressing the spin down hole reflection ( $x=1$ means spin up saturation in the ferromagnet) (see Fig. 2). In the case of $z \neq 0$, we can see that an increase in the barrier potential 


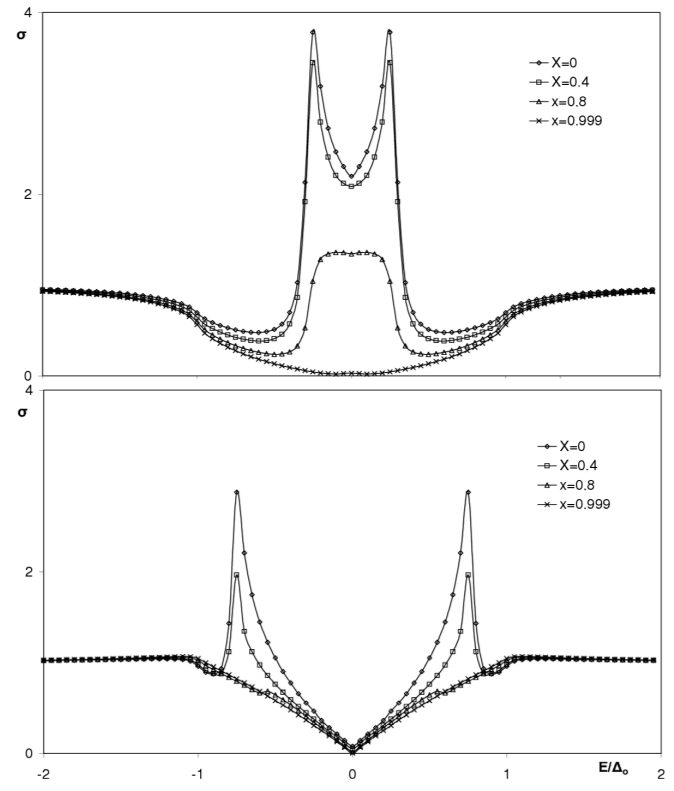

Fig. 3. The normalized tunneling conductance $\mathrm{F} / \mathrm{I} / \mathrm{TS}$ junction with pairing symmetry $B_{1 \mathrm{~g}} \times E_{\mathrm{u}}$ (the top part) and $B_{2 \mathrm{~g}} \times E_{\mathrm{u}}$ (the bottom part) for $z=2.5$ and $\beta=0$.

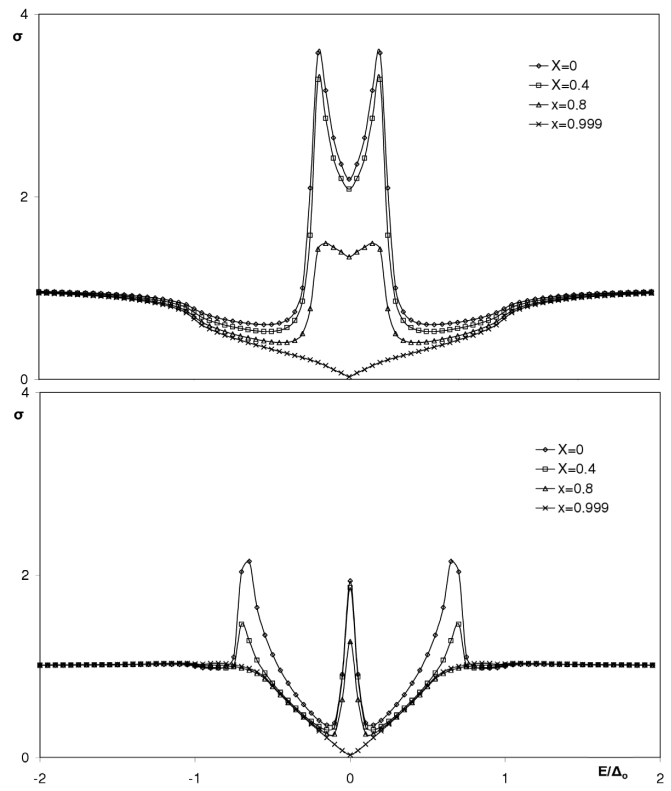

Fig. 4. The normalized tunneling conductance F/I/TS junction with pairing symmetry $B_{1 \mathrm{~g}} \times E_{\mathrm{u}}$ (the top part) and $B_{2 \mathrm{~g}} \times E_{\mathrm{u}}$ (the bottom part) for $z=2.5$ and $\beta=\pi / 16$. 


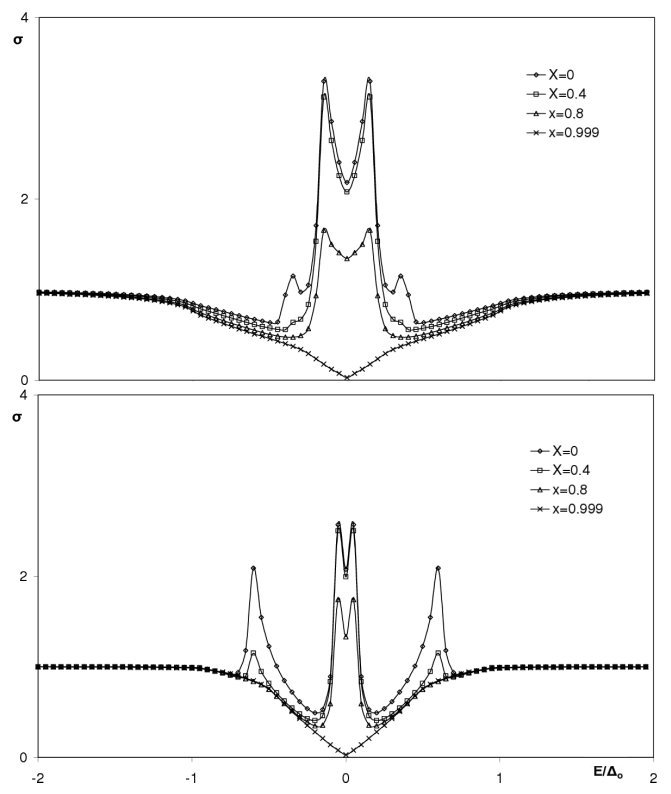

Fig. 5. The normalized tunneling conductance $\mathrm{F} / \mathrm{I} / \mathrm{TS}$ junction with pairing symmetry $B_{1 \mathrm{~g}} \times E_{\mathrm{u}}$ (the top part) and $B_{2 \mathrm{~g}} \times E_{\mathrm{u}}$ (the bottom part) for $z=2.5$ and $\beta=\pi / 11$.

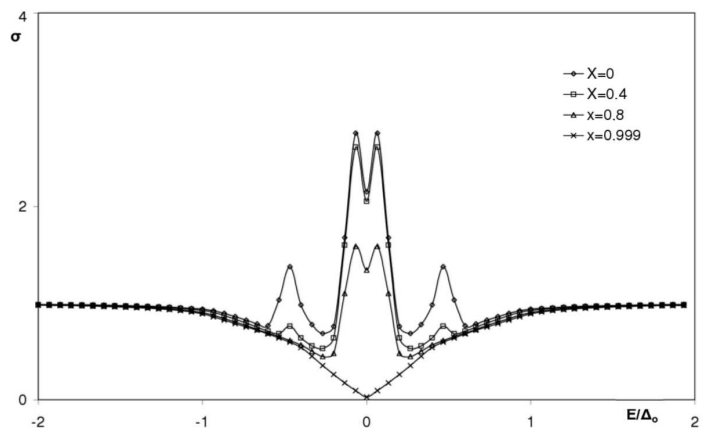

Fig. 6. The normalized tunneling conductance $\mathrm{F} / \mathrm{I} / \mathrm{TS}$ junction with pairing symmetry $B_{1 \mathrm{~g}} \times E_{\mathrm{u}}$ for $z=2.5$ and $\beta=\pi / 8$. The pairing symmetry $B_{2 \mathrm{~g}} \times E_{\mathrm{u}}$ yields the same result.

exhibits the mid-gap peak structure (Figs. 3-7). The resonant tunneling occurs for energy equal to the energy of the bound states determined from the equation $\delta\left(E, \Theta_{s}, \varphi_{+}-\varphi_{-}\right)=0$. The positions of the mid-gap conductance peaks depends both on the phase difference of the pairing potentials felt by ELQ and HLQ and on the angle $\beta$ of the orientation of the interface. The tunnelling conductance spectra plotted in Figs. 3-7 evidently show that the tunnelling spectroscopy is very sensitive to the phases of the pairing potentials and orientation of the interface. Changing $\beta$ from 0 to $\pi / 4$ we can see that: 


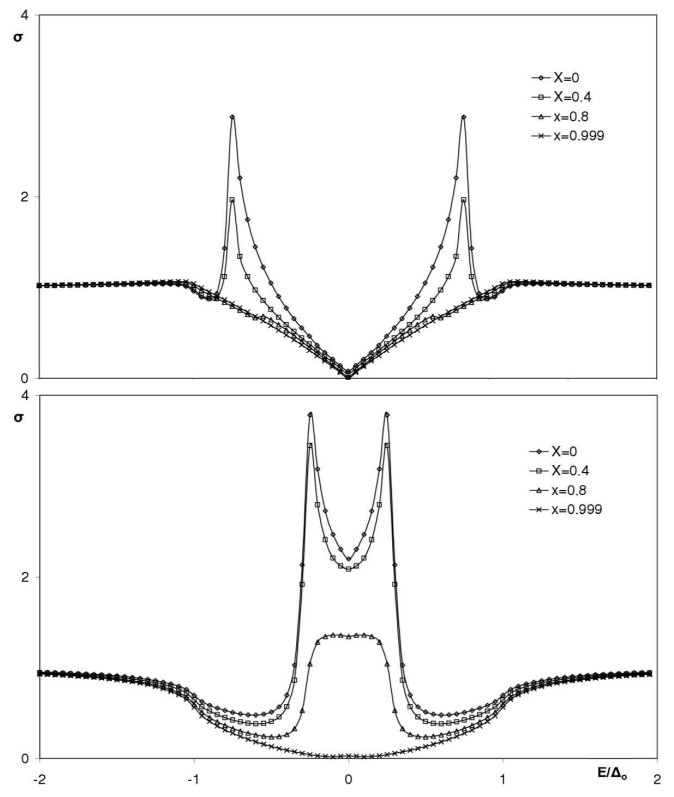

Fig. 7. The normalized tunneling conductance $\mathrm{F} / \mathrm{I} / \mathrm{TS}$ junction with pairing symmetry $B_{1 \mathrm{~g}} \times E_{\mathrm{u}}$ (the top part) and $B_{2 \mathrm{~g}} \times E_{\mathrm{u}}$ (the bottom part) for $z=2.5$ and $\beta=\pi / 4$.

a) for small $\beta$ only two mid-gap peaks appear and in the case of $B_{1 \mathrm{~g}} \times E_{\mathrm{u}}$ state the peaks are localized near the centre of the gap but in the case of $B_{2 \mathrm{~g}} \times E_{\mathrm{u}}$ state they are localized near the edges of the gap;

b) for intermediate values of $\beta$ all four mid-gap peaks emerge;

c) for greater values of $\beta$ again a two-peak structure appears.

The results obtained for $\beta=0$ and $\beta=\pi / 4$ in [2] are reproduced in our paper.

\section{Final remarks}

The resonance tunneling conductance in the $F / I / T S$ junction depends upon:

a) height of the barrier potential;

b) strength of the molecular field in the ferromagnet;

c) phase difference of the pair potentials contained in ELQ and HLQ;

d) orientation of the interface with respect to the crystal axis of the triplet superconductor.

If signs of the pair potentials are opposite, then the zero-energy bound states at the interface are formed. These bound states lead to a zero-bias conductance peak. Increasing strength of the molecular field strongly suppresses the tunnelling conductance. In particular, a zero-bias peak evolves towards a zero bias deep. The finite barrier potentials yield tunnelling conductance spectra with mid-gap peaks. Positions of the mid-gap peaks depend on the phase difference between the pair potentials, contained in ELQ and HLQ, as well as on the orientation of the interface. 


\section{Acknowledgments}

A partial support from the Foundation for Polish Science (FNP) is gratefully acknowledged.

\section{References}

[1] S. Kashiwaya, Y. Tanaka, Rep. Prog. Phys. 63, 1641 (2000).

[2] N. Stefanakis, J. Phys., Condens. Matter 13, 3643 (2001).

[3] N. Yoshida, Y. Tanaka, J. Inoue, S. Kashiwaya, J. Phys. Soc. 68, 1071 (1999).

[4] C. Bergemann, A.P. Mackenzie, S.R. Julian, D. Forsythe, E. Ohmichi, Adv. Phys. 52, 639 (2003).

[5] C. Honerkamp, M. Sigrist, J. Low Temp. Phys. 111, 895 (1998).

[6] G. Litak, J.F. Annett, B.L. Györffy, K.I. Wysokiński, Phys. Status Solidi B 241, 1 (2004).

[7] L. Kowalewski, R.J. Wojciechowski, P. Wojtuś, Phys. Status Solidi B 243, 116 (2006).

[8] G.E. Blonder, M. Tinkham, T.M. Klapwijk, Phys. Rev. B 25, 4515 (1982). 